\title{
Observations of magnetic fields in intracluster medium
}

\author{
Federica Govoni \\ INAF - Osservatorio Astronomico di Cagliari, Strada 54, Loc. Poggio dei Pini, 09012 \\ Capoterra $(\mathrm{Ca})$, Italy \\ email: fgovoni@oa-cagliari.inaf.it
}

\begin{abstract}
The presence of $\mu G$-level magnetic fields associated with the intracluster medium of galaxy clusters is now widely acknowledged. Our knowledge of their properties has greatly improved in the recent years thanks to both new radio observations and the developments of new techniques to interpret data.
\end{abstract}

Keywords. magnetic fields, galaxies: clusters: general, polarization

\section{Radio halos and Faraday rotation measures}

Most of what we know about intracluster magnetic fields derives from the study of radio halos and Faraday rotation measures of polarized radio galaxies located inside or behind galaxy clusters (see e.g. the reviews by Carilli \& Taylor 2002, Govoni \& Feretti 2004, Ferrari et al. 2008, Feretti et al. 2012).

Sensitive radio observations have revealed diffuse emission from the central regions of some merging galaxy clusters. These radio sources, which extend over Mpc scales and are called radio halos, are diffuse, low-surface-brightness, and steep-spectrum synchrotron sources with no obvious optical counterparts. To date, approximately 50 radio halos are known. They demonstrate the existence of relativistic electrons and magnetic fields spread in the intracluster medium. The assumption that radio halos have the magnetic energy density comparable to the energy density in relativistic electrons, requires a volumeaveraged magnetic field $\sim 0.1-1 \mu \mathrm{G}$. Sometimes the intracluster magnetic fields can be ordered on scales of hundreds of kpc, as revealed in A2255 (Govoni \& Feretti 2005, Pizzo et al. 2011) and MACS J0717.5+3745 (Bonafede et al. 2009), where a polarized signal, possibly associated to the radio halo, has been detected. Actually, total intensity and polarization intensity radio halo surface brightness fluctuations are strictly related to the magnetic field power spectrum (Tribble 1991, Murgia et al. 2004). Thus, observations of radio halos have been used to study the structure of the cluster wide magnetic fields by comparing observations with mock halos from turbulent magnetic fields (Murgia et al. 2004, Govoni et al. 2006, Vacca et al. 2010, Xu et al. 2012).

High resolution, detailed RM images of extended cluster radio galaxies have been obtained (e.g. Eilek \& Owen 2002, Taylor et al. 2007, Guidetti et al. 2008, Guidetti et al. 2010, Bonafede et al. 2010, Vacca et al. 2012). These data are usually consistent with central magnetic field strengths of a few $\mu \mathrm{G}$, but stronger fields, with values exceeding $\simeq 10 \mu \mathrm{G}$, are derived in the inner regions of relaxed cooling core clusters. By analyzing the RM of radio galaxies located at different projected distance from the cluster center, it is possible to investigate the radial decrease of the magnetic field with the gas density (e.g. Dolag et al. 2001). The RM distributions seen across the radio galaxies present patchy structures. The observed RM fluctuations indicate that the intracluster 
magnetic field is not regularly ordered but turbulent on scales ranging from tens of kpc to $\lesssim 100$ pc. Dedicated software tools and semi-analytical approach have been developed to constrain the magnetic field power spectrum parameters (Enßlin \& Vogt, Murgia et al. 2004, Laing et al. 2008, Kuchar \& Enßlin 2011). The magnetic field power spectrum can be approximated with a power law with the slope close to the Kolmogorov index in some clusters but shallower index are also observed.

In addition to detailed RM studies focused on single clusters, magnetic fields in galaxy clusters can be investigated statistically. Clarke et al. (2001) analyzed the average RM values as a function of source impact parameter for a sample of Abell clusters. They found a clear broadening of the RM distribution toward small projected distances from the cluster center (see also e.g. Johnston-Hollitt et al. 2004, Govoni et al. 2010), clearly indicating that most of the RM contribution comes from the intracluster medium and proving that magnetic fields are present in all galaxy clusters. Bonafede et al. (2011) selected a sample of massive galaxy clusters and used the NRAO VLA Sky Survey (Condon et al. 1998) to analyze the fractional polarization of hundreds radio sources lying at different projected distances from the cluster center. They detected a clear trend of the fractional polarization, being smaller for sources close to the cluster center and increasing with increasing distance form the cluster central regions. This trend is interpreted as the result of an higher depolarization, occurring because of the higher magnetic field and gas density at the cluster center, and can be reproduced by a magnetic field model with a central value of few $\mu \mathrm{G}$.

\section{References}

Bonafede, A., Feretti, L., Giovannini, G., et al. 2009, A\&A 503, 707

Bonafede, A., Feretti, L., Murgia, M., et al. 2010, A\&A 513, A30

Bonafede, A., Govoni, F., Feretti, et al. 2011, A\&A 530, A24

Carilli, C. L. \& Taylor, G. B. 2002, ARA\& $A$ 40, 319

Clarke, T. E., Kronberg, P. P., \& Böhringer, H. 2001, ApJ 547, L111

Condon, J. J., Cotton, W. D., Greisen, E. W., et al. 1998, AJ 115, 1693

Dolag, K., Schindler, S., Govoni, F., \& Feretti, L. 2001, A\&A 378, 777

Eilek, J. A. \& Owen, F. N. 2002, ApJ 567, 202

Enßlin T. A. \& Vogt C. 2003, A\&A 401, 835

Feretti, L., Giovannini, G., Govoni, F., \& Murgia, M. 2012, A\&SA Rev. 20, 54

Ferrari, C., Govoni, F., Schindler, S., et al. 2008, Space Science Reviews 134, 93

Govoni, F. \& Feretti, L. 2004, Int. J. Mod. Phys. D, Vol. 13, N.8, p. 1549

Govoni, F., Murgia, M., Feretti, L., et al. 2005, A\& $A$ 430, L5

Govoni, F., Murgia, M., Feretti, et al. 2006, A\& $A$ 460, 425

Govoni, F., Dolag, K., Murgia, M., et al. 2010, A\&3A 522, A105

Guidetti, D., Murgia, M., Govoni, F., et al. 2008, A\&A 483, 699

Guidetti, D., Laing, R. A., Murgia, M., et al. 2008, Aछ A 514, A50

Johnston-Hollitt, M., Hollitt, C. P., \& Ekers, R. D. 2004, The Magnetized Interstellar Medium, Eds: B. Uyaniker, W. Reich, and R. Wielebinski p.13

Kuchar, P. \& Enßlin, T. A. 2011, A\& A 529, A13

Laing, R. A., Bridle, A. H., Parma, P., \& Murgia, M. 2008, MNRAS 391, 521

Murgia M., Govoni F., Feretti L., et al. 2004, A\&\&A 424, 429

Pizzo, R. F., de Bruyn, A. G., Bernardi, G., \& Brentjens, M. A. 2011, A\&A 4 525, A104

Taylor G. B., Fabian, A. C., Gentile, G., et al. 2007, MNRAS 382, 67

Tribble, P. C. 1991, MNRAS 253, 147

Vacca, V., Murgia, M., Govoni, F., et al. 2010, A\&A 514, 71

Vacca, V., Murgia, M., Govoni, F., et al. 2012, A\&A 540, 38

Xu, H., Govoni, F., Murgia, M., et al. 2012, ApJ 759, 40 